\title{
Perfil Epidemiológico de Pacientes com Diabetes Tipo 1 Tratados em Hospital Terciário no Sul do Brasil
}

\section{Epidemiological Profile of Patients with Type 1 Diabetes Treated at a Tertiary Hospital in Southern Brazil}

\author{
Renata Soares Carvalho ${ }^{1}$ \\ Rafael Antonio Parabocz ${ }^{2}$ \\ Gianna Carla Alberti Schrut ${ }^{3}$ \\ Ana Claudia Garabeli Cavalli Kluthcovsky ${ }^{4}$ \\ Matheo Augusto Morandi Stumpf ${ }^{5}$
}

\section{RESUMO}

Objetivo: Analisar o perfil clínico e metabólico de pacientes adultos portadores de DM1 em tratamento em um hospital terciário no Sul do Brasil. Metodologia: Estudo descritivo, transversal, realizado através da análise de prontuários dos pacientes entre 2014 a 2018, obtendo-se dados de variáveis epidemiológicas, clínicas e metabólicas. O teste $t$ de Student foi utilizado para as variáveis contínuas com distribuição gaussiana e, para as variáveis não paramétricas, foi aplicado o teste de Wilcoxon O nível de significância adotado foi de $5 \%$. Resultados: Dentre os 60 pacientes estudados, obteve-se uma média de idade de 35,5 anos. Destes, $63,3 \%$ possuíam mais de 10 anos de evolução da doença. Elevou-se de $30,6 \%$ para $64,4 \%$ do total de pacientes que passaram da insulina recombinante humana para análogos de insulina ao longo do tratamento. Ocorreu uma redução estatisticamente significativa nos valores da glicemia de jejum e da hemoglobina glicada, comparando-se com o início do acompanhamento até o atual, assim como na frequência de hipoglicemias. Conclusão: Apesar do controle glicêmico não corresponder às metas estabelecidas, verificou-se uma redução significativa dos valores da hemoglobina glicada com o acompanhamento especializado, em conformidade com outros estudos, que apontam que o controle ideal destes pacientes ainda é um desafio, sendo necessário não apenas o manejo terapêutico, como também propostas de intervenção educacionais e de mudanças de estilo de vida nesses pacientes.

\section{DESCRITORES}

Diabetes Mellitus. Tipo 1. Perfil Clínico. Controle Metabólico.

\begin{abstract}
Introduction: Type 1 diabetes mellitus (DM1) is a disorder characterized by complete deficiency in insulin production. It has great relevance due to high rates of morbidity and mortality, implying direct and indirect costs for the health system. Objective: To analyze the clinical and metabolic profile of adult patients with DM1 undergoing treatment at a tertiary hospital in southern Brazil. Methodology: A descriptive, cross-sectional study, carried out through the analysis of patient records between 2014 and 2018 , obtaining data on epidemiological, clinical and metabolic variables. The Student's t test was used for continuous variables with Gaussian distribution, and for non-parametric variables, the Wilcoxon test was chosen. The level of significance adopted was $5 \%$. Results: Among the 60 patients studied, an average age of 35.5 years was obtained. Of these, $63.3 \%$ had more than 10 years of disease evolution. It rose from $30.6 \%$ to $64.4 \%$ of the total number of patients who switched from recombinant human insulin to insulin analogues during treatment. There was a statistically significant reduction in the values of fasting glucose and glycated hemoglobin, comparing the beginning of the follow-up to the current one, as well as the frequency of hypoglycemia. Conclusion: Although glycemic control does not correspond to the established goals, there was a significant reduction in glycated hemoglobin values with specialized monitoring, in accordance with other studies, which point out that the ideal control of these patients is still a challenge, requiring not only the therapeutic management, but educational intervention proposals and lifestyle changes in these patients.
\end{abstract}

\section{DESCRIPTORS}

Type 1. Diabetes Mellitus. Clinical Profile. Metabolic Control.

\footnotetext{
${ }^{1}$ Acadêmica de Medicina da Universidade Estadual de Ponta Grossa (UEPG), Ponta Grossa, Paraná, Brasil.

${ }^{2}$ Acadêmico de Medicina da Universidade Estadual de Ponta Grossa (UEPG), Ponta Grossa, Paraná, Brasil.

${ }^{3}$ Mestre em Medicina Interna; Professora Adjunta do Curso de Medicina da Universidade Estadual de Ponta Grossa (UEPG), Ponta Grossa, Paraná, Brasil.

${ }^{4}$ Doutora em Medicina Interna e Ciências da Saúde; Professora Adjunta do Curso de Medicina da Universidade Estadual de Ponta Grossa (UEPG), Ponta Grossa, Paraná, Brasil.

${ }^{5}$ Médico pela Universidade Estadual de Ponta Grossa (UEPG), Ponta Grossa, Paraná, Brasil.
} 
$\mathrm{O}$ diabetes mellitus tipo 1 (DM1) é um distúrbio metabólico caracterizado pela deficiência completa na produção de insulina, devido à destruição autoimune ou idiopática das células beta-pancreáticas, resultando em hiperglicemia ${ }^{1,2}$. Estima-se que o DM1 corresponde de 5 a $10 \%$ do total de casos de diabetes em todo mundo e, que mais de 30 mil brasileiros sejam portadores de DM1, sendo que o Brasil ocupa o terceiro lugar em prevalência de DM1 no mundo, segundo a International Diabetes Federation ${ }^{3}$.

O diagnóstico clínico do DM1 é realizado por meio dos sintomas clássicos - poliúria, polidipsia, polifagia e emagrecimento ${ }^{1,4}$. $\mathrm{O}$ início da doença pode ser abrupto, sendo que em muitas vezes a suspeita do diagnóstico é realizada após o quadro de cetoacidose diabética, que pode ser a primeira manifestação da doença em até um terço dos casos $^{4,5}$. Para o diagnóstico laboratorial de DM1 são avaliados a presença dos sintomas clássicos acrescidos de glicemia casual $\geq 200 \mathrm{mg} / \mathrm{dL}^{3}$. O diagnóstico também pode ser realizado a partir da glicemia de jejum, sendo confirmatório quando for $\geq 126 \mathrm{mg} / \mathrm{dL}$. Além disso, pode-se utilizar a hemoglobina glicada (HbA1c), sendo elaborado o diagnóstico caso seja maior ou igual a $6,5 \%{ }^{1,3}$.

Existe uma alta prevalência de complicações nos portadores de DM1 (arteriopatia, nefropatia, neuropatia e retinopatia diabética) $)^{1,3,4}$, inclusive aumento do risco cardiovascular ${ }^{4,6}$. Neste conceito, destaca-se a importância do controle metabólico nesses pacientes. Na prática clínica, a avaliação do controle glicêmico é realizada mediante a utilização dos testes de glicemia e de $\mathrm{HbA} 1 \mathrm{c}$, sendo este último considerado o exame pa- drão-ouro para avaliar o controle metabólico do indivíduo com DM11,3. A Sociedade Brasileira de Diabetes ${ }^{1}$ recomenda como meta níveis de $\mathrm{HbA} 1 \mathrm{c}<7 \%$, visto que há uma relação consistente entre seus níveis aumentados e risco de complicações microvasculares ${ }^{1,4}$.

O tratamento do DM1 visa manter o controle glicêmico dentro dos limites da normalidade, com o objetivo de minimizar o risco de complicações crônicas e melhorar a qualidade de vida de seus portadores. O uso de insulina é imprescindível ao tratamento do DM1, sendo necessária sua instituição desde o início do diagnóstico ${ }^{4}$. Dispõe-se de diversas formas, incluindo a insulina recombinante humana e os análogos de insulina ${ }^{7}$. O esquema intensivo de aplicações diárias, necessita de um controle rigoroso que impacta diretamente na qualidade de $v^{2} a^{7,8}$. Isso exige um processo educativo constante de autocuidado, que deve ser abordado de forma ampla e multidisciplinar ${ }^{9,10}$.

Os baixos índices de sucesso no controle glicêmico dos portadores de DM1 evidenciam a necessidade de um acompanhamento contínuo e integral, levando-se em consideração não apenas o processo da doença em si, mas todo o âmbito psicossocial e o impacto na qualidade de vida desses pacientes ${ }^{8,9}$.

Diante da complexidade dessa doença, das elevadas taxas de morbimortalidade e complicações associadas, torna-se necessário um levantamento de dados acerca do acompanhamento longitudinal do cuidado e do tratamento correto. Assim, o objetivo deste estudo foi analisar o perfil clínico e metabólico de pacientes adultos portadores de DM1 em tratamento em um hospital terciário no Sul do Brasil. 


\section{METODOLOGIA}

Trata-se de um estudo transversal, descritivo e de abordagem quantitativa, realizado por meio da análise de dados dos prontuários de pacientes adultos portadores de DM1 atendidos pelo ambulatório de endocrinologia de um hospital terciário, de ensino e público, localizado na Região Sul do Brasil.

O estudo foi desenvolvido por meio da pesquisa de todos os prontuários classificados pelo CID E10 - diabetes mellitus insulinodependente, obtidos por meio do sistema eletrônico de Gestão Hospitalar e Ambulatorial do SUS (GSUS). A pesquisa estendeu-se também à análise de prontuários físicos, fornecidos pelo Serviço de Arquivo Médico e Estatística do hospital.

Os critérios de inclusão foram determinados a partir do diagnóstico definitivo de DM1 apresentado nos prontuários registrados. Os critérios de elegibilidade foram: I) quadro clínico clássico (poliúria, polidipsia, polifagia e emagrecimento); II) pesquisa de anticorpos positiva; III) uso invariável de insulina desde o início do seu tratamento; IV) abertura do quadro com cetoacidose diabética. Os critérios de exclusão da pesquisa foram: I) pacientes portadores de DM tipo 2 em uso de insulina II) pacientes insulinodependentes que faziam acompanhamento em outras especialidades não relacionadas ao ambulatório de endocrinologia do hospital. Ao todo, foram incluídos neste estudo 60 pacientes, maiores de 18 anos de idade, no período de janeiro de 2014 a dezembro de 2018.

Foram acessados dados epidemio- lógicos da população em estudo referentes à: I) sexo; II) idade; III) cidade de procedência. Também foram coletados dados com relação à procedência do encaminhamento para o serviço de atenção especializada de referência: I) por meio da Unidade Básica de Saúde; II) encaminhamento interno do próprio hospital, seja por outra especialidade ou por entrada pelo pronto-atendimento após quadro de cetoacidose diabética. Também foram coletados dados sobre o tempo decorrido desde o início do acompanhamento, associado à frequência de consultas (relação entre o total meses de acompanhamento e número total de consultas) e acompanhamento em outras especialidades, especialmente aquelas envolvidas diretamente com o manejo do DM1 e suas complicações crônicas associadas - oftalmologia, neurologia, nefrologia, cirurgia vascular e nutricionista.

Em relação ao DM1, foram coletadas informações relacionadas à: I) idade do diagnóstico; II) forma de apresentação ao diagnóstico - sintomas clássicos ou descompensação por cetoacidose diabética; III) modalidade terapêutica ao início do acompanhamento e tratamento atual (NPH associada à insulina regular ou administração de análogos de insulina); IV) esquema utilizado (dose fixa ou contagem de carboidratos); V) presença de sintomas de hipoglicemia; VI) presença de outras doenças autoimunes; VII) pesquisa de autoanticorpos contra as células beta pancreáticas; VIII) presença de complicações (retinopatia, neuropatia, nefropatia, arteriopatia e pé diabético).

As complicações crônicas relacionadas ao diabetes foram consideradas 
da seguinte maneira: I) retinopatia, por confirmação diagnóstica por meio do CID E10.3 (Diabetes mellitus insulinodependente - com complicações oftálmicas); II) neuropatia, por meio de dados do exame físico que corroborassem com sua confirmação (polineuropatia motora/sensitiva ou neuropatia autonômica); III) nefropatia, por meio da medida de microalbuminúria em amostra isolada, sendo que valores acima de $30 \mathrm{mg} / \mathrm{g}$ de creatinina foram adotados como ponto de corte para categorizar nefropatia diabética, associada à dosagem de creatinina; IV) arteriopatia e pé diabético, por meio das descrições nos prontuários e de dados de exame físico registrados.

Outros parâmetros também foram analisados, incluindo: I) comorbidades associadas (presença de hipertensão arterial sistêmica, dislipidemia, tireoideopatia, cardiopatia e depressão): II) medicações de uso contínuo; III) hábitos de vida (etilismo e tabagismo). O índice de massa corpórea (IMC) na primeira e na última consulta foi calculado através da razão peso $(\mathrm{kg})$ pela altura $(m)$ ao quadrado. Além disso, também foram registrados os valores da pressão arterial sistólica (PAS) e pressão arterial diastólica (PAD), na primeira e na última consulta.

Dados laboratoriais também foram igualmente coletados, tanto na primeira quanto na última consulta. Foram adotados os seguintes valores para normalidade, de acordo com a referência adotada pelo hospital: I) glicemia de jejum até $100 \mathrm{mg} / \mathrm{dL}$; II) dosagem de HbA1c até 7\%; III) microalbuminúria em amostra isolada até $30 \mathrm{mg} / \mathrm{g}$ de creatinina; IV) dosagem de creatinina até $1,3 \mathrm{mg} / \mathrm{dL} ; \mathrm{v}$ ) dosagem de hormônio tireoestimulante (TSH) entre 0,30 e 4,20 uUl/ $\mathrm{mL}$; VI) colesterol total menor que $200 \mathrm{mg} / \mathrm{dL}$; VII) LDL abaixo de $100 \mathrm{mg} / \mathrm{dL}$

O teste $t$ de Student para amostras pareadas foi utilizado para as variáveis contínuas com distribuição gaussiana. Para variáveis contínuas não paramétricas, referentes aos dados antropométricos e laboratoriais, foi utilizado o teste de Wilcoxon. $\mathrm{Na}$ avaliação das variáveis categóricas referentes ao tratamento com insulina, foi empregado o teste de $\mathrm{McNe}$ mar para amostras pareadas. Para todos os testes, o nível de significância adotado foi de $5 \%$ ou 0,05 . As demais variáveis categóricas foram descritas por meio de distribuição de frequências absoluta e relativa, enquanto que as variáveis de apresentação contínua foram expressas com medidas de tendência central, amplitude, média e desvio padrão. Todos os dados foram processados no banco de dados Microsoft Excel 2016. A análise estatística foi obtida com auxílio do programa Statistical Package for Social Science (SPSS), versão 18.0.

O estudo foi aprovado pela Comissão Científica teve início após parecer do Comitê de Ética em Pesquisa (CAAE: 07812619.0.0000.0105, parecer CEP $3.183 .539)$.

\section{RESULTADOS}

Este estudo contemplou um total de 60 pacientes com DM1, com idade igual ou superior a 18 anos. Destes, 35 (58,3\%) eram do sexo feminino e $25(41,6 \%)$ eram do sexo masculino. A média de anos de idade dos pacientes foi de $35,5 \pm 12,1$ anos, sendo que apenas 21 (35\%) apresentavam idade infe- 
rior a 30 anos. Com relação ao município de procedência, $22(36,6 \%)$ pertenciam a cidade sede do estudo, enquanto que a maioria $(63,3 \%)$ era procedente de outros municípios. De acordo com o tipo de encaminhamento para o ambulatório, $34(56,6 \%)$ pacientes foram referenciados pela Unidade Básica de Saúde, enquanto 26 (43,3\%) pertenciam ao encaminhamento interno do próprio hospital.

Com relação ao tempo desde o diagnóstico do DM, $38(63,3 \%)$ pacientes possuíam mais de 10 anos de evolução da doença. A idade ao diagnóstico foi mais prevalente entre os 10 a 20 anos (35\%), seguida de 20 a 30 anos (31,6\%). Com relação à forma de apresentação ao diagnóstico, a queixa de emagrecimento foi referida em $41,7 \%$ dos pacientes, seguida de cetoacidose diabética como primeira manifestação da doença em $31,6 \%$ dos pacientes. Polidipsia foi referida em $31,6 \%$ dos pacientes, enquanto poliúria e polifagia foram relatadas em $23,3 \%$ e $18,3 \%$ dos pacientes, respectivamente. A dosagem de autoanticorpos foi constatada em apenas $11,6 \%$ dos pacientes.

De acordo com a história clínica de doenças associadas, $40 \%$ eram portadores de dislipidemia, seguido de $35 \%$ por hipertensão arterial sistêmica, 30\% depressão, $16,6 \%$ tireoideopatia e $13,3 \%$ de cardiopatia. Em relação presença de outras doenças autoimunes, a tireoidite de Hashimoto esteve presente em $15 \%$ dos pacientes, seguida de artrite reumatoide em $5 \%$, doença celíaca em $3,3 \%$, e vitiligo, esclerodermia, e insuficiência adrenal em $1,6 \%$ dos pacientes. O uso de medicação de uso contínuo foi de $71,6 \%$ para até 3 medicações diárias e de $28,3 \%$ para 4 ou mais medicações. O tabagismo foi constatado em $11,6 \%$ dos pacientes e o etilismo em $20 \%$ dos pacientes. A Tabela 1 resume as variáveis sociodemográficas e clínicas da amostra estudada.

A Tabela 2 apresenta a comparação entre as variáveis no início do acompanhamento e o tratamento atual. Os resultados foram estatisticamente significativos nas variáveis tipo de tratamento, esquema de insulina e presença de hipoglicemia. Verificou-se que a maioria dos pacientes iniciaram o tratamento com NPH associado a insulina regular $(69,4 \%)$ e que essa situação se inverteu no tratamento atual, onde a maioria fazia o uso de análogos de insulina (64,4\%). Em relação ao esquema de insulina, $68,3 \%$ dos pacientes faziam o esquema de dose fixa ao início do acompanhamento, valor que se elevou para $83,3 \%$ no tratamento atual. Em relação a ocorrência de hipoglicemia, 58,7\% relataram o sintoma no início do acompanhamento, em comparação a $36,9 \%$ no tratamento atual.

A Tabela 3 mostra as variáveis referentes às complicações decorrentes do DM. Verificou-se que mais da metade dos pacientes relataram episódio de descompensação aguda por cetoacidose diabética em algum momento da doença. Em relação aos desfechos crônicos, $30 \%$ apresentaram neuropatia diabética, seguida por retinopatia diabética $(28,3 \%)$, nefropatia diabética $(25 \%)$, e, por fim, o pé diabético e amputação apresentaram a mesma incidência (5\%) para a população estudada.

A Tabela 4 demostra a comparação entre as variáveis clínicas e laboratoriais, no início do acompanhamento e no tratamento atual. Ocorreu diferença estatisticamente significativa apenas nas variáveis IMC, PAS, glicemia plasmática de jejum, dosagem de HbA1c, dosagem de microalbuminúria em 
Tabela 1. Distribuição das variáveis sociodemográficas e clínicas para o total de pacientes com diabetes mellitus tipo1

\begin{tabular}{|c|c|c|}
\hline Variáveis & $\mathrm{n}(60)^{*}$ & $\%$ \\
\hline \multicolumn{3}{|l|}{ Idade dos pacientes } \\
\hline Menos de 30 anos & 21 & 35,0 \\
\hline 30 anos ou mais & 39 & 65,0 \\
\hline \multicolumn{3}{|l|}{ Sexo } \\
\hline Feminino & 35 & 58,3 \\
\hline Masculino & 25 & 41,6 \\
\hline \multicolumn{3}{|l|}{ Município } \\
\hline Cidade sede do estudo & 22 & 36,6 \\
\hline Outras cidades & 38 & 63,3 \\
\hline \multicolumn{3}{|l|}{ Tipo de encaminhamento } \\
\hline Unidade Básica de Saúde & 34 & 56,6 \\
\hline Encaminhamento interno do próprio hospital & 26 & 43,3 \\
\hline \multicolumn{3}{|l|}{ Tempo de diabetes (em anos) } \\
\hline Até 10 anos & 22 & 36,6 \\
\hline Mais de 10 anos & 38 & 63,3 \\
\hline \multicolumn{3}{|l|}{ Idade ao diagnóstico do DM } \\
\hline Até 10 anos & 08 & 13,3 \\
\hline De 10 a 20 anos & 21 & 35,0 \\
\hline De 20 a 30 anos & 19 & 31,6 \\
\hline Acima de 30 anos & 12 & 20,0 \\
\hline \multicolumn{3}{|l|}{ Forma de apresentação } \\
\hline Cetoacidose diabética & 19 & 31,6 \\
\hline Emagrecimento & 25 & 41,7 \\
\hline Poliúria & 14 & 23,3 \\
\hline Polidipsia & 19 & 31,6 \\
\hline Polifagia & 11 & 18,3 \\
\hline História familiar diabetes & 23 & 38,3 \\
\hline Tabagismo & 07 & 11,6 \\
\hline Etilismo & 12 & 20,0 \\
\hline \multicolumn{3}{|l|}{ Número de medicamentos uso contínuo } \\
\hline Até 3 & 43 & 71,6 \\
\hline 4 ou mais & 17 & 28,3 \\
\hline Dislipidemia & 24 & 40,0 \\
\hline Hipertensão arterial & 21 & 35,0 \\
\hline Depressão & 18 & 30,0 \\
\hline Tireoideopatia & 10 & 16,6 \\
\hline Cardiopatia & 08 & 13,3 \\
\hline Doença autoimune & 16 & 26,6 \\
\hline
\end{tabular}

${ }^{*}$ Obs.: A soma dos pacientes pode não totalizar a amostra de 60 devido a dados faltantes ou indisponíveis. 
Tabela 2. Distribuição das variáveis referentes ao tratamento com insulina, para o total de pacientes com diabetes mellitus tipo1

\begin{tabular}{l|c|c|c|c|c}
\hline \multirow{2}{*}{ Variáveis } & \multicolumn{2}{c|}{ Início do tratamento } & \multicolumn{2}{c}{ Tratamento Atual } & \multirow{2}{*}{$p$} \\
\cline { 2 - 5 } & $\mathrm{n}$ & $\%$ & $\mathrm{n}$ & $\%$ & \\
\hline Tipo de tratamento & & & & & \\
\hline NPH + Regular & 41 & 69,4 & 21 & 35,5 & $<0,001$ \\
\cline { 2 - 5 } $\begin{array}{l}\text { Análogos de insulina } \\
\text { Esquema de insulina }\end{array}$ & 18 & 30,6 & 38 & 64,4 & \\
\hline $\begin{array}{l}\text { Dose fixa } \\
\text { Contagem de carboidratos }\end{array}$ & 41 & 68,3 & 50 & 83,3 & $<0,01$ \\
\cline { 2 - 5 } Hipoglicemia & 19 & 31,6 & 10 & 16,6 & \\
\hline Sim & & & & & \\
Não & 27 & 58,7 & 17 & 36,9 & 0,01 \\
\hline
\end{tabular}

Utilizado Teste McNemar para amostras pareadas.

Tabela 3. Distribuição das variáveis referentes às complicações decorrentes do diabetes mellitus tipo 1, para o total de pacientes analisados

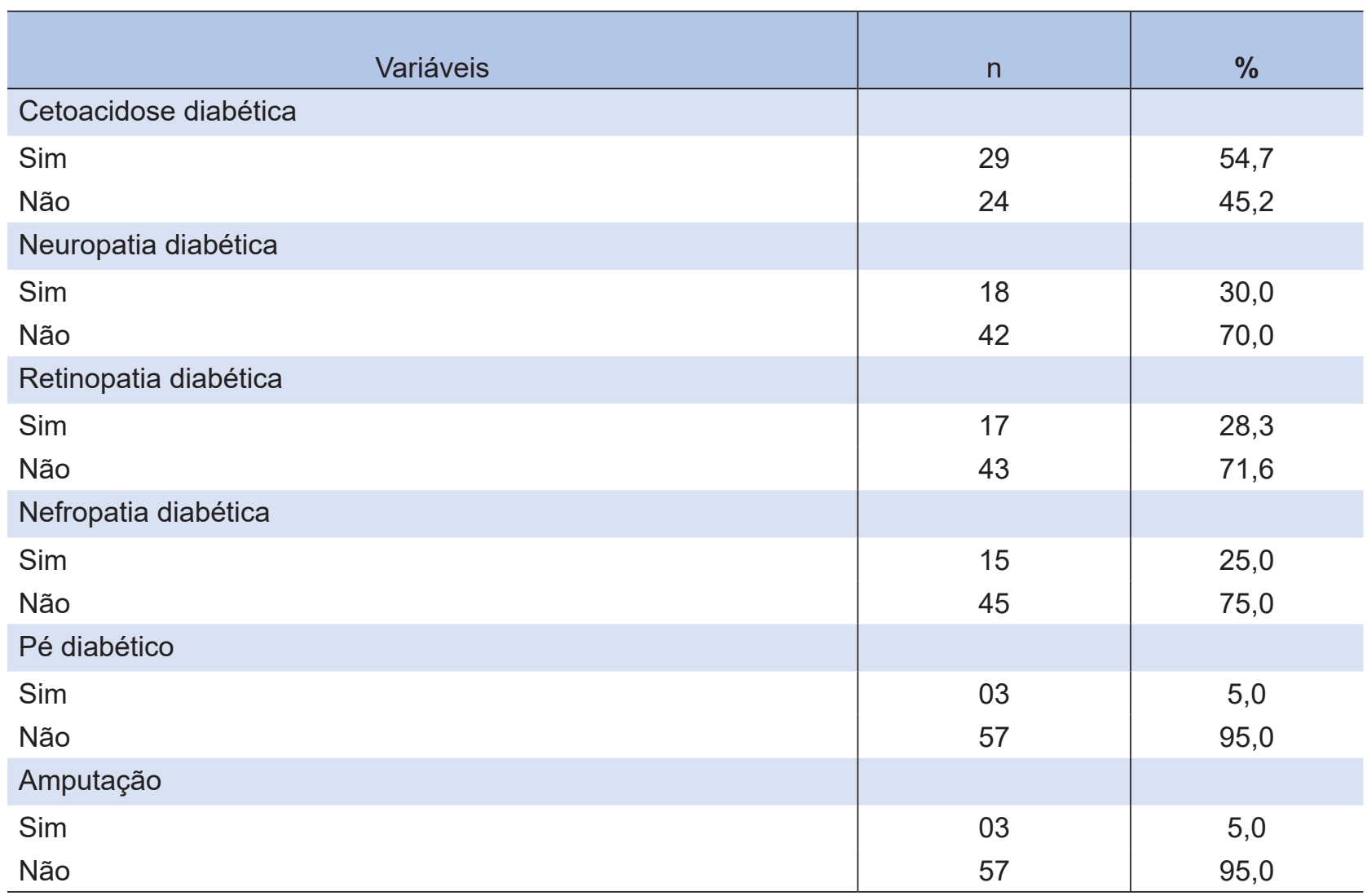


Tabela 4. Comparação entre as variáveis clínicas e laboratoriais

\begin{tabular}{|c|c|c|c|c|c|}
\hline \multirow{2}{*}{ Variáveis } & \multicolumn{2}{|c|}{ Início do tratamento } & \multicolumn{2}{|c|}{ Tratamento atual } & \multirow[b]{2}{*}{$p$} \\
\hline & $\begin{array}{l}\text { Média } \\
\text { (desvio- } \\
\text { padrão) }\end{array}$ & $\begin{array}{c}\text { Mediana } \\
\text { (Min-Máx) }\end{array}$ & $\begin{array}{l}\text { Média } \\
\text { (desvio- } \\
\text { padrão) }\end{array}$ & $\begin{array}{c}\text { Mediana } \\
\text { (Min-Máx) }\end{array}$ & \\
\hline $\begin{array}{l}\text { Índice de massa corpórea } \\
\left(\mathrm{IMC}-\mathrm{kg} / \mathrm{m}^{2}\right)\end{array}$ & $23,6(4,4)$ & $23,7(14,3-4,8)$ & $25,3(4,07)$ & $25,7(16,9-35,5)$ & $<0,001^{*}$ \\
\hline $\begin{array}{l}\text { Pressão arterial sistólica } \\
(\mathrm{mmHg})\end{array}$ & $114,7(17,3)$ & $110(90-160)$ & $122,8(16,14)$ & $120(90-160)$ & $<0,01^{\text {** }}$ \\
\hline $\begin{array}{l}\text { Pressão arterial diastólica } \\
(\mathrm{mmHg})\end{array}$ & $72,9(11,4)$ & $70(50-120)$ & $74,1(10,5)$ & $70(60-110)$ & $0,40^{* *}$ \\
\hline $\begin{array}{l}\text { Glicemia plasmática de } \\
\text { jejum (mg/dL) }\end{array}$ & $256,3(98,0)$ & $238(80-508)$ & $187,3(88,3)$ & $171(53-454)$ & $<0,001^{* *}$ \\
\hline Hemoglobina glicada (\%) & $10,9(2,3)$ & $10,2(6,9-6,5)$ & $9,7(2,1)$ & $9,4(6,3-14,9)$ & $0,001^{* *}$ \\
\hline $\begin{array}{l}\text { Microalbumina (mg/g de } \\
\text { creatinina) }\end{array}$ & $19,9(22,1)$ & $12,2(0,75-84)$ & $107,2(262,6)$ & $17(3-1302)$ & $0,02^{* *}$ \\
\hline Creatinina (mg/dL) & $0,8(0,3)$ & $0,8(0,3-2,6)$ & $0,8(0,6)$ & $0,71(0,3-4,99)$ & $0,05^{\star *}$ \\
\hline $\mathrm{TSH}(\mathrm{uUl} / \mathrm{mL})$ & $3,0(2,5)$ & $2,5(0,07-14,4)$ & $2,8(2,2)$ & $2,25(0,8-10,7)$ & $0,81^{* *}$ \\
\hline Colesterol Total (mg/dL) & $177,2(42,7)$ & $167(99-300)$ & $186,6(47,6)$ & $170(118-329)$ & $0,76^{\star *}$ \\
\hline $\mathrm{HDL}(\mathrm{mg} / \mathrm{dL})$ & $47,2(13,1)$ & $47(24-76)$ & $48,0(13,9)$ & 47 (25-79) & $0,91^{*}$ \\
\hline LDL (mg/dL) & $103,9(33,2)$ & $97(51-183)$ & $108,9(42,6)$ & $101(39-227)$ & $0,85^{\star *}$ \\
\hline Triglicerídeos (mg/dL) & $119,3(73,1)$ & $96,5(42-380)$ & $158(122,7)$ & $119(46-624)$ & $0,10^{* *}$ \\
\hline
\end{tabular}

amostra isolada e dosagem de creatinina. As demais variáveis não obtiveram resultados estatisticamente significativos.

A Tabela 5 representa a distribuição das variáveis referentes ao acompanhamento no ambulatório de endocrinologia. A maioria possuía tempo de acompanhamento superior a dois anos. Em relação à frequência das consultas, notou-se que a maioria possuía periodicidade com um intervalo maior que 3 meses. Do total de pacientes avaliados $(n=60), 35 \%$ abandonaram o acompanhamento no ambulatório em questão.

\section{DISCUSSÃO}

Os achados epidemiológicos da po- pulação em estudo apontam que não existiu predileção significativa por sexo, o que condiz com outros estudos ${ }^{1,11}$. A idade média dos pacientes no momento do estudo foi de 35,5 anos e o tempo desde do diagnóstico de DM foi, em sua maioria, maior que 10 anos de evolução. Embora uma grande parte dos estudos nesta área abordem a faixa etária de crianças e adolescentes ${ }^{11-14}$, no presente estudo a amostra foi constituída em sua totalidade por indivíduos adultos, maiores de 18 anos, os quais começaram a ser acompanhados em hospital especializado com o diagnóstico já estabelecido e manejo terapêutico prévio.

Com relação à idade no momento do diagnóstico, são relatados na literatura dois picos de incidência: entre 4 a 5 anos de idade 
Tabela 5. Distribuição das variáveis referentes ao acompanhamento no ambulatório de endocrinologia, para o total de pacientes com diabetes mellitus tipo1

\begin{tabular}{l|c|c}
\hline \multicolumn{1}{c|}{ Variáveis } & $n$ & $\%$ \\
\hline Tempo de acompanhamento & 25 & 41,6 \\
\hline Até 2 anos & 35 & 58,3 \\
2 anos ou mais & & \\
\hline Frequência de consultas* & 12 & 26,0 \\
A cada 3 meses & 34 & 73,9 \\
3 meses ou mais & & \\
Acompanhamento em outras especialidades & 32 & 53,3 \\
Oftalmologia & 05 & 8,3 \\
Nefrologia & 05 & 8,3 \\
Vascular & 05 & 8,3 \\
Neurologia & 10 & 16,6 \\
Psiquiatria & 06 & 10,0 \\
Nutricionista & 13 & 21,6 \\
Outra especialidade & & \\
\hline Abandono do acompanhamento no ambulatório & 21 & 35,0 \\
\hline Sim & 39 & 65,0 \\
\hline Não
\end{tabular}

*Foram considerados para cálculo de frequência de consultas apenas os pacientes que Acompanharam por no mínimo 6 meses no ambulatório, desta forma, a amostra reduziu-se para n=46.

e aos 10 a 14 anos $^{11}$, sendo que este último coincide com a população em estudo, onde foi predominante o diagnóstico entre os 10 a 20 anos de idade. Isso pode estar relacionado ao início da puberdade e um consequente aumento da demanda por insulina, o que causaria uma sobrecarga pancreática, induzindo ao início da doença ${ }^{11}$. Além disso, o segundo grupo de maior incidência foi entre 20 e 30 anos, o que corrobora com diversos estudos acerca da forma lentamente progressiva do DM1, o chamado latent autoimmune diabetes in adults (LADA), que afeta adultos jovens e cursam com uma forma mais bran- da da doença ${ }^{4,15}$. No estudo de Trinajstic et al. ${ }^{16} 82,9 \%$ dos casos de DM1 foram diagnosticados até os 30 anos de idade ${ }^{16}$.

O diagnóstico precoce do DM1 depende de vários fatores, incluindo a capacidade de percepção dos sintomas pelo paciente e/ ou família, até que se faça o diagnóstico definitivo. Infelizmente, a falta de procura pelos serviços de saúde relaciona-se com altas taxas de apresentação da doença em estado de cetoacidose diabética, que culminam com a procura por atendimento apenas durante a descompensação estabelecida ${ }^{5}$. No presente estudo, o DM1 foi diagnosticado em um epi- 
sódio de descompensação em $31,7 \%$ dos pacientes, corroborando com outros estudos que mostram que até um terço dos diagnósticos acontecem apenas nessas circunstâncias ${ }^{4,5}$.

De acordo com a literatura, quase a totalidade dos casos de DM1 são de etiologia autoimune e em menos de $5 \%$ a causa é dita como idiopática ${ }^{12}$. O autoanticorpo mais utilizado com a finalidade diagnóstica no portador adulto de DM1 é o antidescarboxilase do ácido glutâmico (anti-GAD), que está presente em $80 \%$ dos casos de DM1 de instalação recente ${ }^{2,4,17}$. Porém, neste estudo, apenas uma pequena porcentagem de pacientes realizou a dosagem de anticorpos, devido a não gratuidade deste exame pelo Sistema Único de Saúde.

Pacientes com DM1 representam uma população de alto risco para desenvolvimento de doenças cardiovasculares e mortalidade precoce $^{6}$. Segundo Orchard ${ }^{18}$, indivíduos com DM1 têm um risco dez vezes maior de eventos cardiovasculares do que as populações não diabéticas de mesma idade ${ }^{18}$. Em um estudo multicêntrico, realizado em centros de atenção secundária e terciária no Brasil ${ }^{19}$, foi constatado que a porcentagem de pacientes que não se enquadravam nas metas pressóricas e lipídicas estabelecidas chegava em torno de 40 a $50 \%$, o que corrobora com a importância do controle metabólico nestes pacientes, devido a evidências de que o gerenciamento intensivo de peso, colesterol e pressão arterial são eficazes na diminuição do risco de complicações nestes pacientes. Em nosso estudo, $40 \%$ dos pacientes eram portadores de dislipidemia e $35 \%$ de hipertensão arterial sistêmica.

O clássico estudo prospectivo Diabetes Control and Complications (DDCT) ${ }^{20}$ apontou o esquema intensivo com múltiplas aplicações diárias de insulina, como o padrão ouro no tratamento do DM1, reduzindo, comprovadamente, suas complicações crônicas associadas, além de redução significativa da $\mathrm{HbA}_{1} \mathrm{c}^{20}$. Entretanto, o maior obstáculo para a adesão desse esquema terapêutico é o aumento da frequência de episódios de hipoglicemia, o que dificulta a adesão ao tratamento ${ }^{8}$.

No presente estudo, foi constatado diminuição significativa na frequência de episódios de hipoglicemia, comparando-se o início do acompanhamento e o tratamento atual, e isto pode ser explicado porque a maioria da amostra estudada iniciou com o uso de insulina NPH associada à insulina regular e grande parte destes pacientes migrou para o uso de análogos de insulina ao longo do tratamento. Existem diversos estudos comparativos que avaliaram que a principal diferença nesses dois tipos de tratamento associa-se com a menor frequência de eventos hipoglicêmicos com o uso de análogos de insulina, enquanto que a redução dos níveis glicêmicos e valores de HbA1c são pouco significativos ${ }^{21-22}$.

No DM1, a abordagem mais efetiva no controle da glicemia pós-prandial é o esquema de contagem de carboidratos, que possui a flexibilidade de ajuste conforme a alimentação ${ }^{11,22}$. Neste estudo, observou-se uma diminuição significativa do número de pacientes que utilizavam a contagem de carboidratos, ao longo do tratamento $(p<0,01)$. Acredita-se que a baixa adesão a este esquema pode estar relacionada com o nível de entendimento e de aconselhamento nutricional desses pacientes, o que corrobora com outros estudos ${ }^{11}$, apesar das variáveis socioeconômicas e do nível de escolaridade não terem sido abordadas no presente artigo. Importante também ressaltar, que esse esquema não interfere apenas no 
controle glicêmico, mas na qualidade de vida desses pacientes ${ }^{22}$.

De acordo com a Sociedade Brasileira de Diabetes, é recomendado como meta níveis de $\mathrm{HbA} 1 \mathrm{c}$ menores que $7 \%$, visto que existe uma relação consistente entre seus níveis aumentados e risco de complicações ${ }^{1,4}$. Em relação ao controle glicêmico, observou-se uma redução estatisticamente significativa dos valores de glicemia de jejum e de $\mathrm{HbA} 1 \mathrm{c}$ $(p<0,001)$, em relação ao início e o tratamento atual. No entanto, esses valores ainda não correspondem à meta de controle glicêmico estabelecida, semelhante ao encontrado em outros estudos ${ }^{11,19,22}$. Gomes et al. ${ }^{19}$ em estudo multicêntrico nacional realizado em adultos portadores de DM1, demonstraram $\mathrm{HbA} 1 \mathrm{c}$ acima dos valores esperados em aproximadamente $80 \%$ dos pacientes, evidenciando o baixo controle glicêmico, mesmo quando atendidos em serviços de atenção secundária ou terciária ${ }^{19}$.

A dosagem de HbA1c deve ser realizada pelo menos duas vezes por ano em pacientes com controle glicêmico estável e dentro das metas terapêuticas estabelecidas ${ }^{7}$. Em contraposição, naqueles que não atingem o controle adequado, a avaliação deve ser mais frequente, sendo recomendado a cada 3 a 4 meses $^{4,7}$. No ambulatório onde foi realizado o estudo, constatou-se que a frequência de consultas foi menor do que o recomendado, considerando o controle glicêmico inadequado desses pacientes. Esse fator pode ter grande influência na adesão, visto que a não periodicidade de avaliação do paciente interfere na adequação do tratamento e ajuste de doses, de importância ímpar no acompanhamento do paciente com DM1.

Cabe ressaltar que este estudo de- tém limitações, por tratar-se de um estudo transversal, por meio da revisão de prontuários físicos e eletrônicos, onde não foi possível obter todas as variáveis integralmente na população em estudo. Entretanto, reflete também resultados positivos, pois permite conhecer o perfil da realidade local, e, assim, traçar estratégias específicas de intervenção, pois a terapêutica farmacológica unicamente não garante a estabilidade da doença. Assim, devem ser propostas intervenções educacionais e comportamentais, para obtenção das metas glicêmicas e prevenção de complicações, que estão amplamente relacionadas com o aumento de morbimortalidade dessa população. Uma das possíveis ações a serem empreendidas é a implementação de uma equipe multidisciplinar para promoção de ações educativas, com foco no entendimento do paciente acerca de sua doença, da importância da adesão ao tratamento e sua complexidade, apoio nutricional referente à contagem de carboidratos e implementação de dieta adequada, além do cuidado integral e prevenção de comorbidades associadas.

\section{CONCLUSÃO}

Embora este estudo seja referente um ambulatório de atenção terciária e uma grande parte dos pacientes dispusesse de esquemas terapêuticos adequados, a maioria deles manteve-se com controle glicêmico incompatível com as metas estabelecidas, em conformidade com outros estudos, que apontam que o controle ideal destes pacientes ainda é um desafio, sendo necessário não apenas o manejo terapêutico, mas propostas de intervenção educacionais e de mudanças de estilo de vida. 


\section{REFERÊNCIAS}

1. Sociedade Brasileira de Diabetes (SBD). Classificação e diagnóstico do diabetes mellitus. Diretrizes da Sociedade Brasileira de Diabetes 2017-2018; São Paulo: Editora Clannad 2017

2. Silva MER, Mory D, Davini E. Marcadores genéticos e auto-imunes do diabetes melito tipo 1: da teoria para a prática. Arq Bras Endocrinol Metab. 2008; 52(2):166-180.

3. Internacional Diabetes Federation (IDF). Diabetes Atlas. 7th ed. Brussels, Belgium: International Diabetes Federation; 2015.

4. Junior RMM, Almeida SL, Forti A, Gusmão A, Vilar L. Diabetes Mellitus: classificação e diagnóstico. In Vilar L (et al.). Endocrinologia Clínica. 6. Ed. Rio de Janeiro: Guanabara Koogan, 2016; p.617-30.

5. Souza LCVF, Kraemer GC, Koliski A, Carreiro JE, Cat $\mathrm{MNL}$, et al. Cetoacidose diabética como apresentação inicial de diabetes tipo 1 em crianças e adolescentes: estudo epidemiológico no sul do Brasil. Rev. Paul. Pediatr. 2020; 38: e 2018204:1-8

6. González L, Chertkoff A, Burgos M, Triffone L, Sanabria $\mathrm{H}$, et al. Diabetes mellitus tipo 1 y enfermedad cardiovascular. Rev. Soc. Argent. Diabetes. 2020; 54 (2):71-90

7. Ministério da Saúde. Protocolo clínico para dispensação de análogos de insulina para pacientes com Diabetes Mellitus Tipo 1 na rede pública de saúde do Paraná. Secretaria de Estado da Saúde do Paraná (SESA). [acesso em: 2019Jun 13].

8. Souza RR, Marquete VF, Vieira VCL, Ficher MJB, Spigolon DN, Marcon SS. Cuidado domiciliar à criança e ao adolescente com diabetes mellitus tipo 1 na perspectiva do cuidador. Rev enferm UERJ. 2020; 28:e46013: 1-6

9. La Banca RO, Sparapani VC, Bueno M, Costa T, Carvalho EC, Nascimento LC. Strategies to educate young people with type 1 diabetes mellitus on insulin therapy: systematic review. Texto \& Contexto enferm. 2020; 29:1-21.

10. Batista AFM, Nóbrega VM, Gomes GLL, Santos MM, Fernandes LTB, Collet N. Gestão do Diabetes Tipo 1: necessidades de autocuidado apoiado na transição para adolescência. Saude e pesqui. Impr. 2020; 13 (2):363375.

11. Pereira DA. Perfil clínico-epidemiológico dos pacientes portadores de diabetes mellitus tipo 1 atendidos no ambulatório de endocrinologia pediátrica do Hospital Universitário Polydoro Ernani de São Thiago. Florianópolis, 2009.44p. [acesso em: 2019Jun 04].

12. Pellicciari CR, Camargo LA, Nigri AA, Novo NF. Perfil clínico e laboratorial de pacientes pediátricos com diabetes mellitus tipo 1, atendidos em um hospital público terciário de Sorocaba, São Paulo, e sua relação com a adesão ao tratamento. Fac Ciênc Méd Sorocaba. 2017;19(2):61-66.
13. Victório VMG, Andrade ALM, Silva AMB, Machado WL, Enumo SRF. Adolescentes com Diabetes Mellitus tipo 1. Saude e Pesqui. 2019; 12(1):63-75.

14. Vargas, DM, Andrade BB, Bork B. Perfil clínico e epidemiológico de crianças e adolescentes com diabetes mellitus 1 atendidos na atenção secundária em Blumenau -SC. Arq. Catarin Med. 2016; 45(3):58-70.

15. Naik RG, Brooks WBM, Palmer JF. Latent autoimmune diabetes in adults..J Clin Endocrinol Metab. 2009; 94(12):4635-44.

16. Trinajstic E, Cicchitti A, González J, Bertona C, Guntsche $Z$, et al. Diabetes mellitus tipo 1: edad de comienzo y presencia em familiares de primer grado. Rev. Soc. Argent. Diabetes. 2020; 54(1):15-20.

17. Tuomi T. Type 1 and type 2 diabetes: what do they have in common? Diabetes. 2005; 54(suppl 2):40-45.

18. Orchard TJ, Costacou T, Kretowski A, Nesto RW. Type 1 diabetes and coronary artery disease. Diabetes Care. 2006; 29:2528-2538.

19. Gomes MB, Coral M, Cobas RA, Dib SA, Canani LH, et al. Prevalence of adults with type 1 diabetes who meet the goals of care in daily clinical practice: A Nationwide multicenter study in Brasil. Diabetes Research and clinical practice. 2012; 97:63-70.

20. The Diabetes Control and Complications Trial/Epidemiology of Diabetes Interventions and Complications (DCCT/ EDIC) Study Research Group. Intensive diabetes treatment and cardiovascular disease in patients with diabetes type 1. N Engl J Med. 2005; 353: 2643-53.

21. Hermansen K, Fontaine P, Kukolja KK, Peterkova V, Leth $G$, et al. Insulin analogues (insulin detemir and insulin aspart) versus traditional human insulins (NPH insulin and regular human insulin) in basal-bolus therapy for patients with type 1 diabetes. Diabetologia. 2004; 47(4):622-629.

22. Guzatti, PR, Balthazar, APS, Canalli MH, Machado TF Fatores associados ao controle glicêmico em pacientes portadores de diabetes mellitus tipo 1. Arq. Catarin Med. 2017; 46(2):26-38.

\section{CORRESPONDÊNCIA}

Matheo Augusto Morandi Stumpf

Av. Carlos Cavalcanti, 4748, Departamento de Medicina,

Uvaranas CEP: 84030-900, Ponta Grossa, Brasil.

E-mail: matheoaugusto@hotmail.com 